\title{
A novel design for a spectropolarimeter; SPEX
}

\author{
A.L. Verlaan, H. van Brug*, and H. Visser \\ TNO Delft, Stieltjesweg 1, 2628 CK Delft, the Netherlands
}

\begin{abstract}
A novel design for a earth observation combined spectrometer and polarimeter is presented. The goal of the instrument is to measure both intensity (radiance) and the state of polarization. Some backgrounds for this instrument are presented but the main part of this article will be on the optical design and the ideas behind it.
\end{abstract}

Keywords: Spectrometer, Aerosols, Cloud detection, Polarimeter

\section{INTRODUCTION}

We present a novel design for SPEX (Spectropolarimeter for Planetary Exploration), an Earth orbiting, high altitude spectropolarimeter for the measurement of aerosol type and concentrations. Purpose of the instrument is to measure both the spectral radiance and the polarization state (degree and angle of linear polarization) of Solar scattered light. As the degree of linear polarization is extremely sensitive to the microphysical properties of atmospheric or surface particles, this instrument can yield valuable information of these particles in the Earth's atmosphere. In previous studies ${ }^{1,2,3}$ an instrument was realized, targeted for operation on a Mars orbiter. Various experiments confirmed the instruments functionality and assessed its accuracy. This new design aims at measurements of the Earth's atmosphere. Core aspects in the instrument design are the absence of moving parts, the use of proven technology and a flexible and modular design. As a result an instrument is designed, based on a push broom spectrometer with a 30degree field of view and a $1.25 \mathrm{~km}$ by $1.12 \mathrm{~km}$ instantaneous ground pixel. Spectropolarimetric measurements are performed over a wavelength range from $375 \mathrm{~nm}$ to $850 \mathrm{~nm}$ with a predicted measurement accuracy of better than $10^{-3}$ on the Degree Of Linear polarization.

By combining several modules, each with its own design, as explained in the design section, a wide range of observation angles (elevation) is obtained that allows for cloud height measurements and distinction between wet and dry aerosols. Wet aerosols show a narrow and clear angular distribution (rainbow effect), whereas dry aerosols yield a broader and smoother angular distribution. To enhance the cloud height measurement a separate module for $\mathrm{O}_{2} \mathrm{~A}$ detection can be added. This module is not included in this paper but it can be stated that this module is a high resolution spectrometer dedicated for the $\mathrm{O}_{2} \mathrm{~A}$ channel.

Activities presented are performed in close cooperation with the SPEX-team, constituting of TNO, SRON, Leiden University, EADS Dutch Space, Cosine, Mecon and are financially supported by the Netherlands Space Organization.

*eddy.vanbrug@tno.nl, +31(0)888668055

Polarization Science and Remote Sensing VI, edited by Joseph A. Shaw, Daniel A. LeMaster, Proc. of SPIE

Vol. 8873, 88730K · C 2013 SPIE · CCC code: 0277-786X/13/\$18 · doi: 10.1117/12.2024083 


\section{DESIGN CONSIDERATIONS}

A large set of instrument requirements is determined in close collaboration with the SPEX team, combining the instruments scientific goals with a minimum instrument complexity. The spectropolarimeter designed should have a swath field of view of at least 30 degrees and multiple viewing directions covering a flight field of view of 110 degrees. This large angular range in the flight direction is required since this allows the observation of the rainbow. The original optical design covered both directions of the field of view in a single module and on a single detector. Due to the anticipated stray light issues this approach was abandoned and a switch was made to a modular design approach, i.e. adding more angles in flight direction should be as simple as adding an extra module. The stray light levels should be low, and the spectral resolution should be such that

$$
\frac{\Delta \lambda}{\lambda} \geq 50
$$

over the full wavelength range from 400 to $800 \mathrm{~nm}$. Key requirement in the design is the required accuracy on the Degree of Linear (DoLP) of 0.001 $\pm 0.005 \cdot$ DoLP.

The optical system consists of three main parts: a telescope, the polarization encoder and a spectrometer. The polarization encoder design is based on a Lyot stage comprising a quarter waveplate followed by two retarder plates. When a polarizer is placed behind the retarder plates, incident light with intensity $\mathrm{I}_{\mathrm{in}}$ results in a signal $\left(\mathrm{I}_{\text {out }}\right)$ that oscillates as a function of wavelength $(\lambda)$. By inserting a polarizer with it pass direction perpendicular to the first one, a signal is obtained that is exactly out of phase to the first one. These signals are describes as:

$$
I_{\text {Out }}=\frac{1}{2} I_{\text {in }}\left[1 \pm D o L P \cdot \cos \left(\frac{2 \pi \delta(\lambda)}{\lambda}+2 \cdot A o L P\right)\right]
$$

Where $\delta(\lambda)$ is the waveplate retardation and AoLP is the Angle of Linear Polarization. Adding these two signals gives the radiance while the ratio between the minima and maxima in the signal is a measure for the degree of linear polarization.

The first element in the polarization encoder optics is a quarter waveplate, which results in equal signals for the two mutually perpendicularly polarized beam components. This scheme works for all states of polarization except for circularly polarized light, which is not an issue since it is known that light scattered by aerosols and clouds will not be circularly polarized.

For a spectrometer design a dispersive element is required. There are basically two options, a prism or a grating. We have chosen for the prism option for several reasons:

1) The transmission can be very high, no strong polarization dependence.

2) Full coverage of the $400-800 \mathrm{~nm}$ range that for a grating would result in order overlap.

3) More equally spaced 'fringes' in the signal as a function of wavelength owing to the glass dispersion.

A key choice is selection of the detector since many design parameters depend on it. We have selected a relatively simple 512x512 detector with square $13 \mu \mathrm{m}$ pixels as a baseline. This detector allows for the imaging of two images of $215 \mathrm{~km}$ swath each (@400km) over 172 pixels. There will be a 30 pixel gap between the two images on the detector. In the spectral direction each image will be spread out over 430 pixels, covering the $375-850 \mathrm{~nm}$ wavelength range. The two images on the detector pertain to the two mutually orthogonal polarization states.

By having a pupil as first element in the telescope optics the sensitivity to instrument pollution in space is strongly reduced. The telescope lenses are fused silica lenses with an AR coating. These AR coatings are optimized to be as polarization independent as possible. 


\section{OPTICAL DESIGN}

The resulting optical system consists of three main parts: a telescope, the polarization encoder and a spectrometer, which are assembled in this order. Figure 1 shows the resulting optical design.

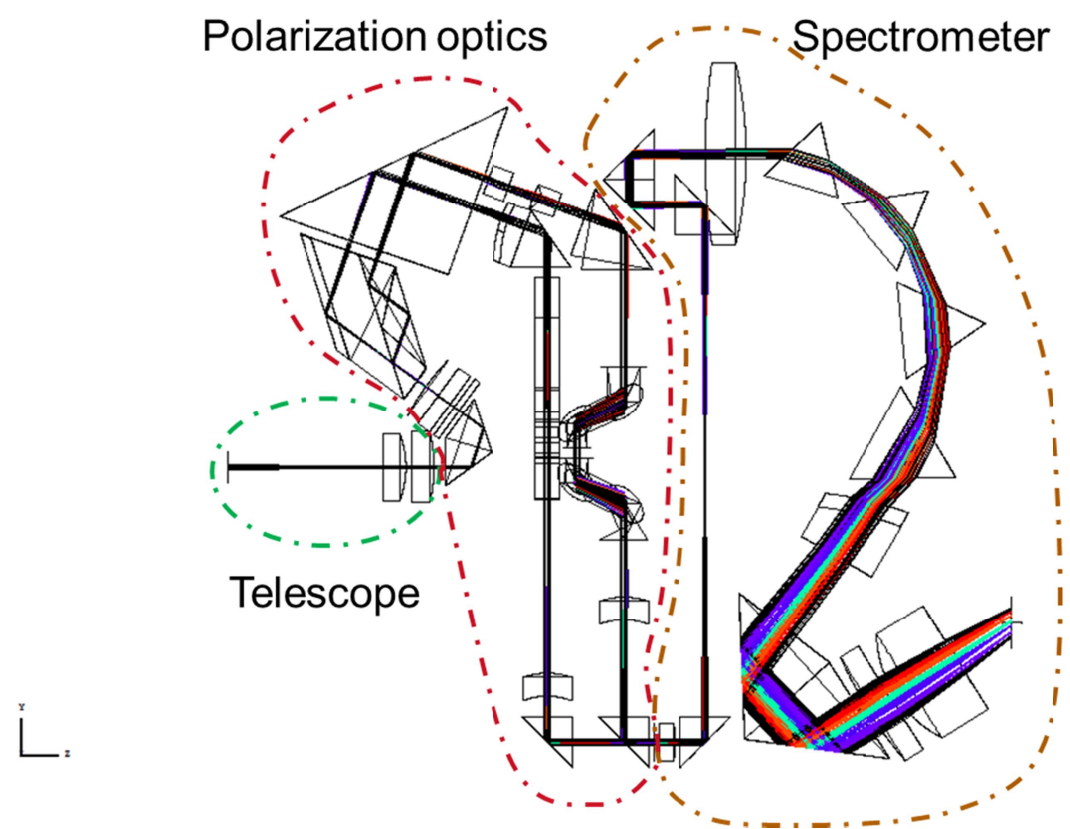

Figure 1: Spectropolarimeter optical scheme and the three main building blocks: the telescope, polarization optics and the spectrometer.

Figure 2 shows the telescope optics in some more detail. The pin-hole design telescope has been designed such that the beams entering the polarization optics are telecentric. Telecentricity is required for the quarter wave prism to reduce the FOV dependence. The quarter wave plate is a fused silica prism with a single thin layer deposited to it to arrive at a phase shift between s- and p-polarization of 45 degrees. By using two reflections in series, the phase shift of 90 degrees, that is needed for a quarter wave function, is realized.

The two retarders are made of crystalline $\mathrm{Al}_{2} \mathrm{O}_{3}$ and $\mathrm{MgF}_{2}$, respectively. Directly behind the entrance slit a polarizing beam splitter is positioned. This component splits the two polarizations and has two exits that are parallel to each other.

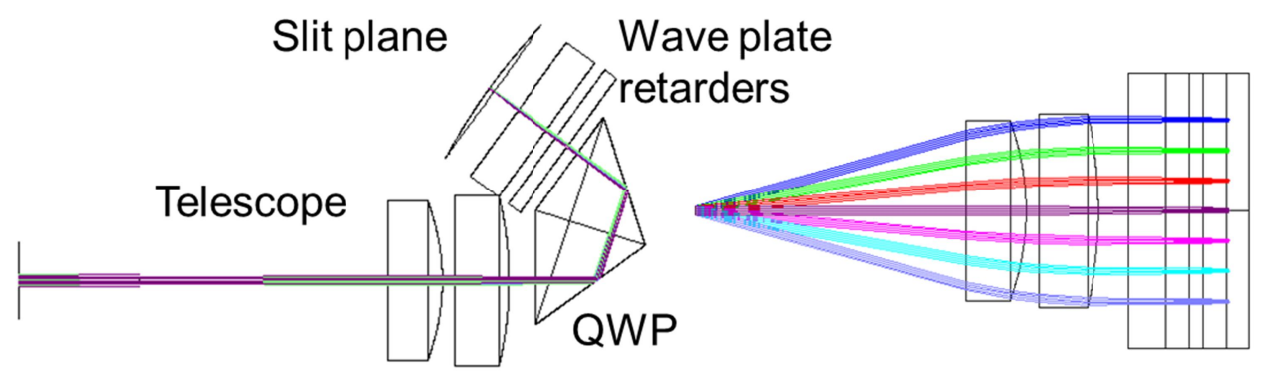

Figure 2: Left: Telescope plus quarter wave prism and the two retarder plates. The final surface is the curved entrance slit. Right: side view showing the across track Field of View

Following the beam path behind the entrance slit we see the polarizing beam splitter with its two exits, an internal reflection mirror used to set the observation angle in flight direction, see figure 3. By applying this folding mirror it is possible to stack multiple channels easily, with their detectors in a single plane, while being highly flexible in the selected viewing angles. By changing the angle in the total internal reflection prism the angle of viewing can be selected. 
The optical systems of the different channels are identical except for this internal reflection prism. The total internal reflection prism has been dimensioned such that the full range of 0 to 55 degrees can be obtained. For the negative viewing angles the module can be flipped over.

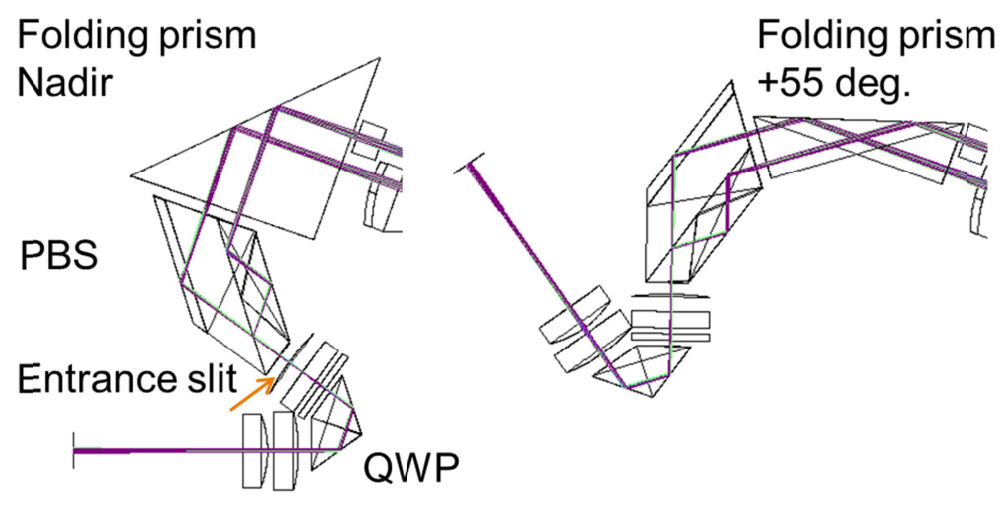

Figure 3: The optical path, comprising the front section of the polarization optics for Nadir viewing (on the left) and 55degree forward viewing (right)

Next is the polarization handling optics, which rotates and aligns the $\mathrm{s}$ and $\mathrm{p}$ light on the spectrometer entrance slit. First the s-polarized path is guided through a halve wave plate under 45 degrees such that it is converted into p-polarized light. The p-polarized path is flipped up-side-down via a three reflections prism. In this way fields can be guided through the spectrometer optics in the same way, which leads to an easier data handling since the co-registration can be made better. After the polarization handling optics the two paths are directed to the spectrometer where the two beams are separated such that the slit images are shifted in the length of the slit. The polarization handling optics are not essential for the basic operation of the instrument, however the added symmetry in the 2 slit images greatly enhances the expected measurement accuracy.

The spectrometer optical design is shown below in figure 4 .

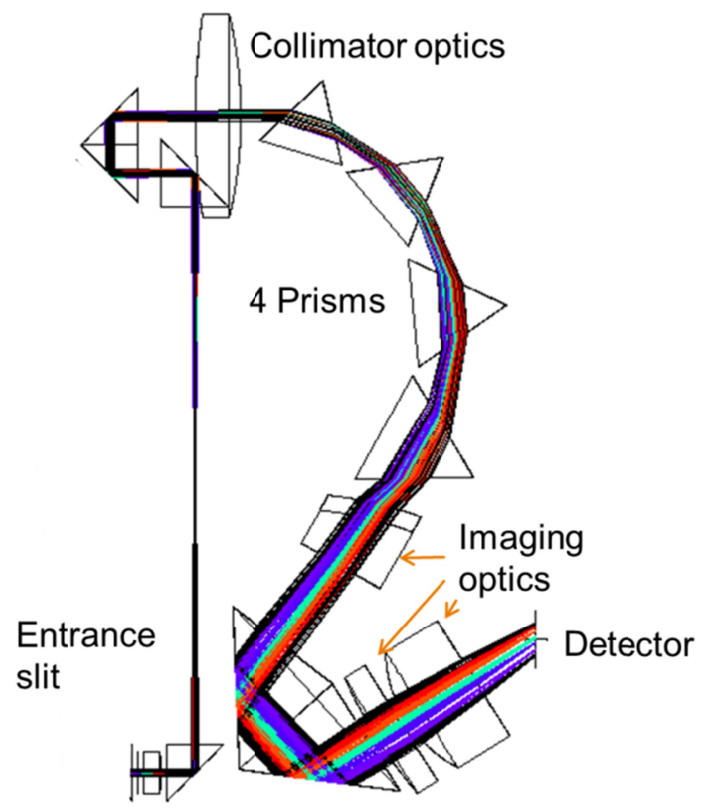

Figure 4: Prism based spectrometer from entrance slit to detector 
After the collimator optics four prisms are used to create a strong enough dispersion. Finally the two dispersed beams are imaged onto the detector that is placed under a large angle, thereby reducing the risk of ghost images due to reflections by the detector.

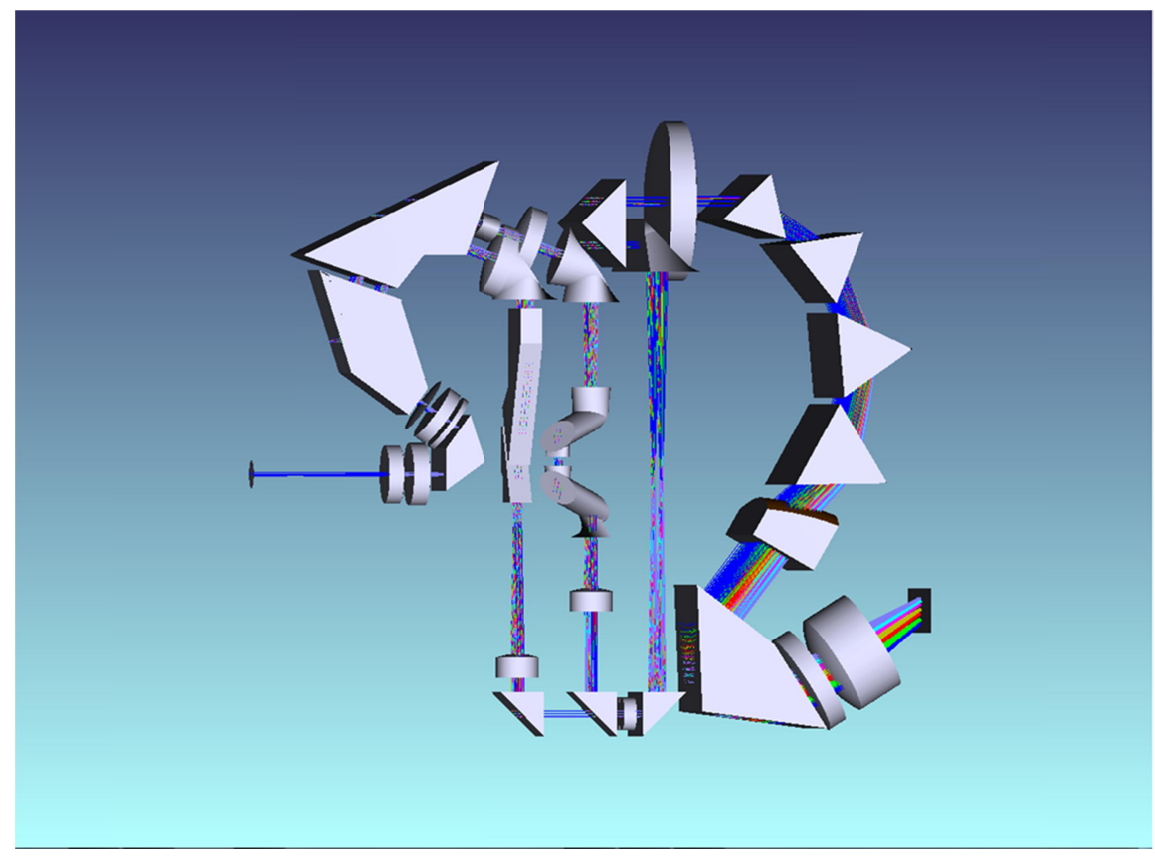

Figure 5: 3D view of the system. The dispersed spectrum onto the detector can clearly be seen.

\section{PERFORMANCE}

The system performance is determined primarily by the AoLP accuracy, as this is the prime parameter of interest for the determination of the aerosol concentration. However a detailed analysis of its error contributors is beyond the targeted scope of this paper. Several additional instrument parameters determine the manufacturability and the usability of the instrument. These comprise spectral straightness, spot sizes and co-registration between the s- and p-polarized signals.

Figure 6 shows one of the two polarization components imaged onto the detector. The other polarization component is imaged onto the other half of the detector and is the mirror image of the shown one. In the horizontal direction of detector in figure 6 the (instantaneous) field of view is imaged. This image is scanned across the Earths' surface as the instrument flies in orbit. In the vertical direction the light is dispersed into a spectrum. 


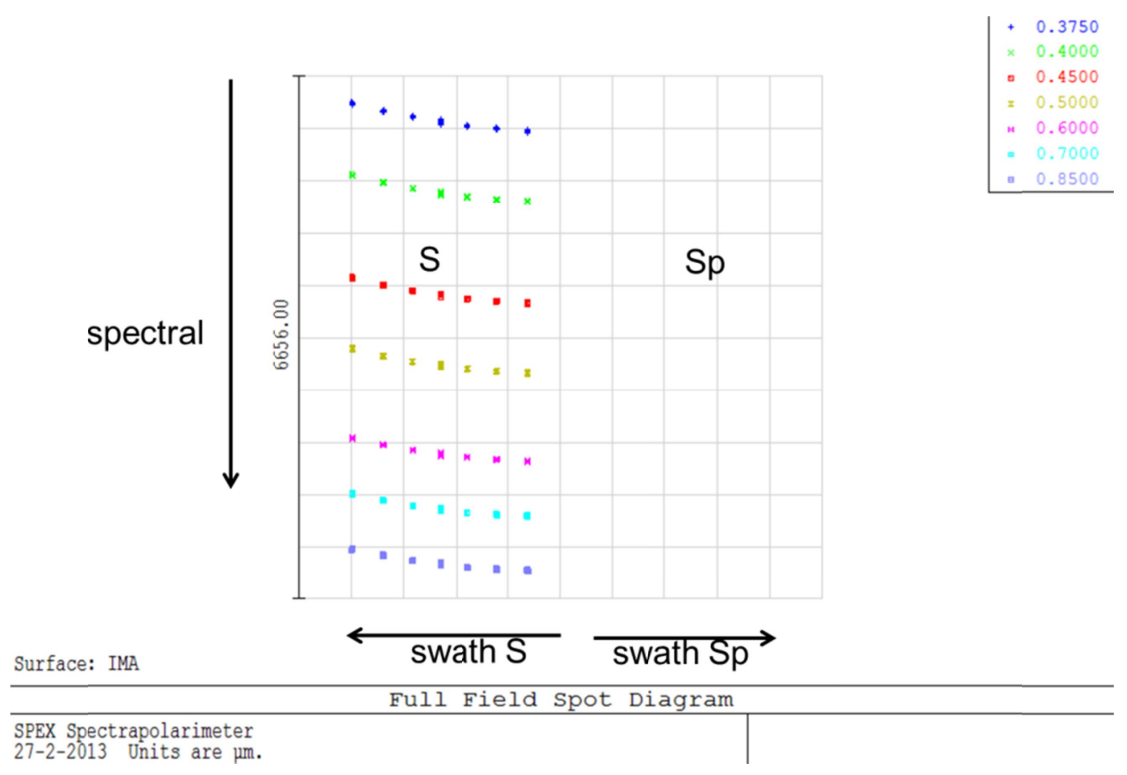

Figure 6: One of the two polarization components on the detector. The other component is the mirror image of this component. Care was taken to have each field component on the same pixel column (for all wavelengths).

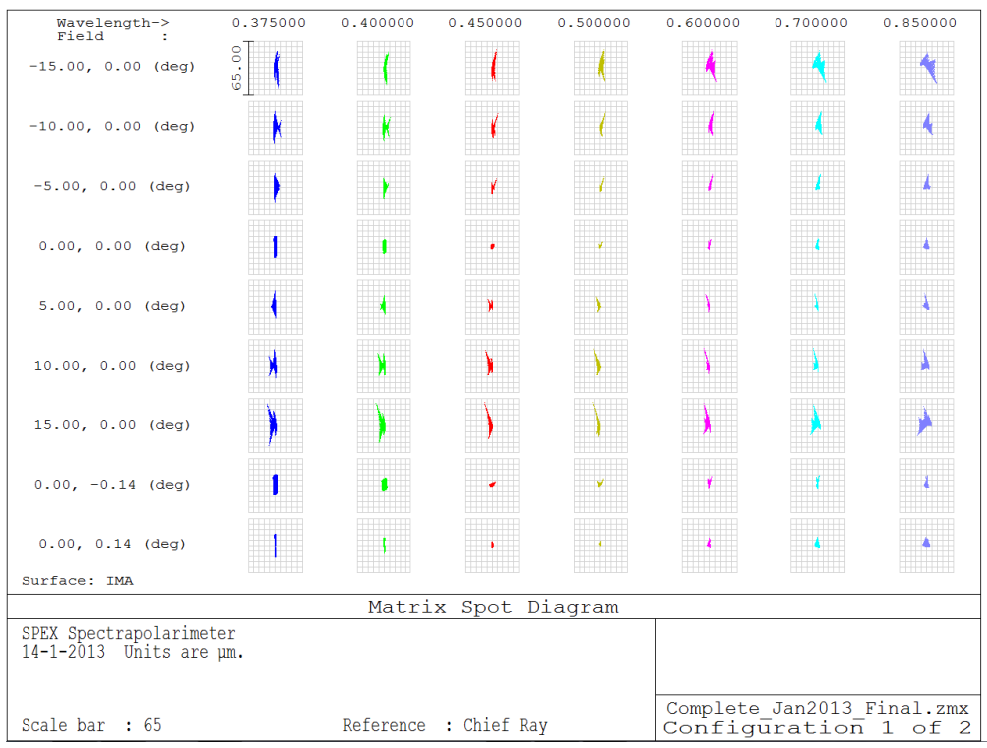

Figure 7: Spot diagram at the detector. Spot images are shown for several fields (vertical) and wavelengths (horizontal)

The spot diagrams on the detector plane are given in Figure 7. As can be seen, the image quality is optimized for spectral resolution while in the spatial direction the spot is still well within 2.5 pixel. The Airy disks are comparable in size with the geometrical spot size. Spot sizes and hence the image quality were analyzed during tolerance analysis. For placement tolerance a value of $0.1 \mathrm{~mm}$ was found and for the angular setting $0.1^{\circ}$. It is expected that the co-registration will prove to be a more stringent requirement for tolerancing. Here more detailed analysis is planned to further the assess the resulting element and positioning accuracies. 
The spectral resolution requirement for the DoLP $(\Delta \lambda / \lambda=50)$ implies that at lower wavelengths more dispersion is required. By applying prisms, rather than a grating, in the spectrometer, this is achieved.

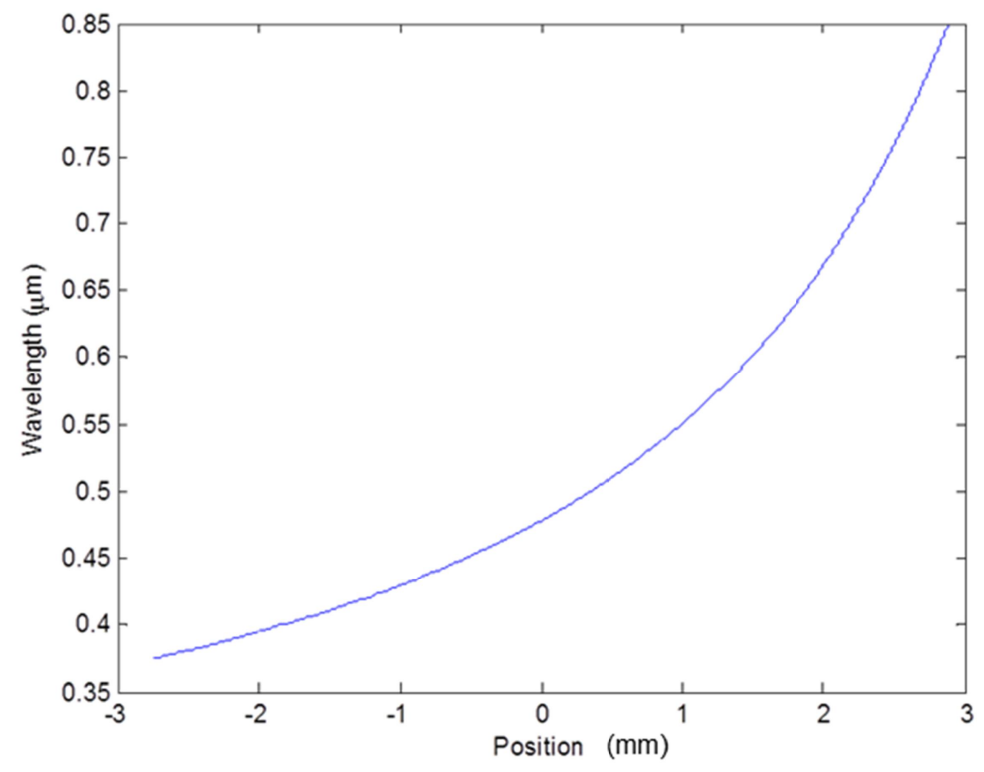

Figure 8: Wavelength as a function of detector position in $\mathbf{m m}$

The positive effect of the use of a prism as dispersive element is shown in figure 8, where the wavelength is depicted as a function of the position on the detector chip. The dispersion is strongest at the lower wavelength side of the spectrum, there where the requirement on spectral resolution is also highest. Owing to this dispersion effect the number of pixels per fringe at $400 \mathrm{~nm}$ is sixteen and reduces to eight pixels per fringe at $800 \mathrm{~nm}$. This number can be adjusted by varying the retarder plates thicknesses.

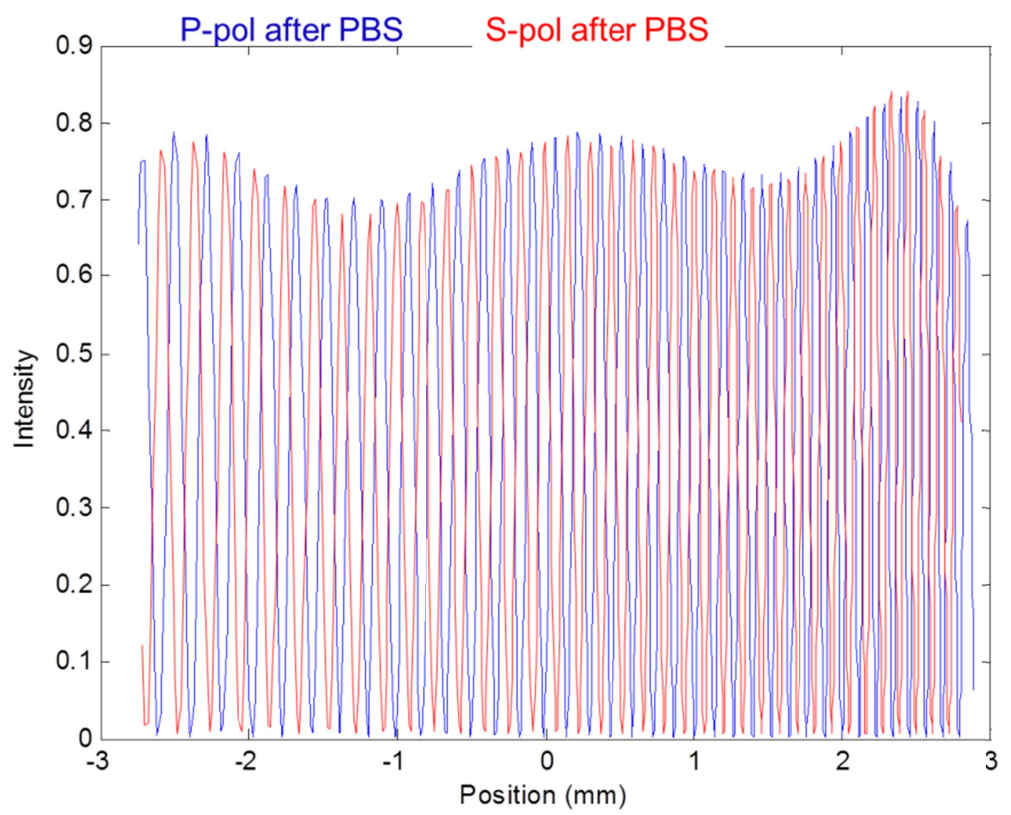

Figure 9: Signal at the detector for $100 \%$ polarized light as input. P-polarized component in blue, S-polarized component in red 
Figure 9 shows the corresponding s- and p-polarized signals on the two detector halves. For analysis purpose they are overlapped in a single plot. It can be seen that the fringes are exactly out of phase, i.e. where in one channel the signal is maximum, the other channel is at a minimum and vice versa. Variation of the fringe density can be seen clearly, wide spacing at the low wavelength side and increasing towards the higher wavelengths. The waviness of the graphs is the result of all the coatings in the design, resulting in some spectral transmission variation. Intensities shown are the actual intensity to be expected at the detector in case of a flat input spectrum.
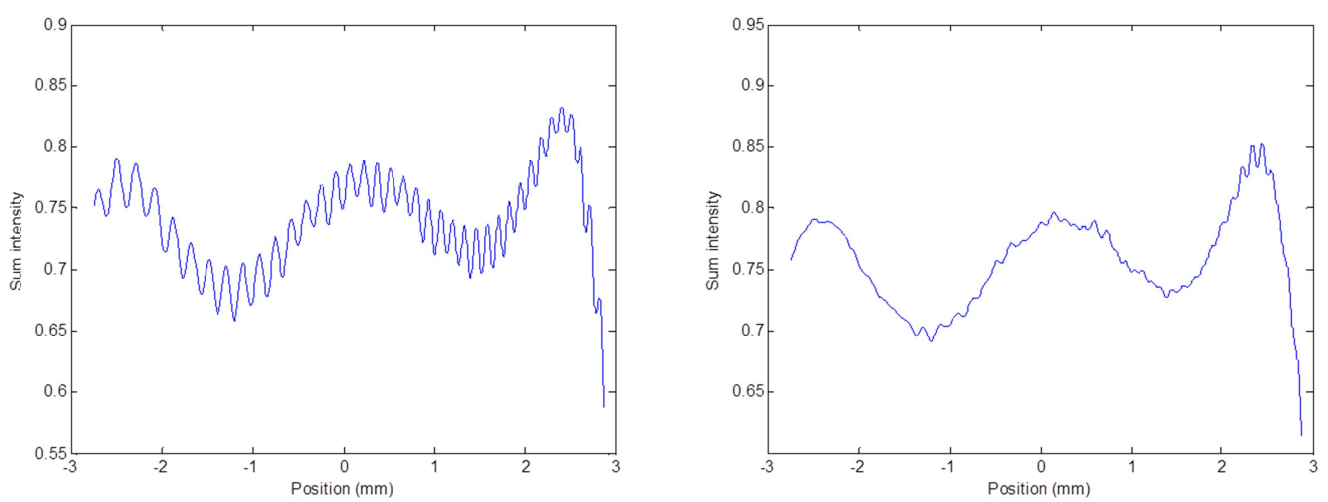

Figure 10: Radiance data obtained by direct addition of the two intensities as shown in Fig.9. For the right hand side graph the two intensities were corrected separately for transmission differences to reduce the wiggle as seen in the left hand side graph.

Direct addition the two polarization states is not sufficient to obtain the radiance data. This is demonstrated in figure 10, the left hand side graph. The wiggles that are visible are due to the fact that the transmission of the system is not truly polarization insensitive. Besides that there are also parts where the two polarization components follow different paths. To remove these wiggles, the two components should be corrected for transmission differences before they are added. This is shown in the right hand side graph of figure 10. Here one component is multiplied by 1.05 as a first order correction test. The result is acceptable for the lower wavelengths. In the actual instrument this scaling factor should be determined during a calibration campaign and as a function of wavelength, i.e. pixel row.

\section{DISCUSSION AND CONCLUSIONS}

The presented SPEX instrument is novel in design and shows a possible performance that exceeds previous designs in terms of stray-light, transmission and measurement accuracy. Yet the resulting design uses only fused silica as glass (except for the waveplates) and relatively simple detectors. Its main features are the modular design and its robustness against pollution and stray light. The design has a number of locations to insert oversized apertures to block scattered light. Tolerances based on the image quality as requirement the system are easily realizable. For placement tolerance a value of $0.1 \mathrm{~mm}$ was found and for the angular setting $0.1^{\circ}$. It is expected that the requirement for wavelengths pertaining to the same ground pixel to fall in the same pixel columns (spectral straightness) and that the two polarization states should have the same wavelength in the same row (co-registration), will lead to tighter requirements for alignment and production. Additional analysis is foreseen for the coming months.

The designed SPEX instrument is a step forward to the understanding of cloud formation and the influence of aerosol particles to the weather. Besides the here presented wide band polarimeter unit, also a high resolution $\mathrm{O}_{2} \mathrm{~A}$ channel has been designed that is modular in design. Adding the $\mathrm{O}_{2} \mathrm{~A}$ channel to the SPEX will provide detailed and valuable information on the cloud height.

\section{REFERENCES}

[1] Rietjens, J.H.H.; Snik, F.; Stam, D.M.; Smit, J.M.; van Harten, G.; Keller, C.U.; Verlaan, A.L.; Laan, E.C.; ter Horst, R.; Navarro, R.; Wielinga, K.; Moon, S.G.; Voors, R. (2010). "SPEX: The spectropolarimeter for planetary exploration.” International Conference on Space Optics 4-8 October 2010 Rhodes, Greece 
[2] Voors, Robert; Moon, Scott G.; Hannemann, Sandro; Rietjens, Jeroen H. H.; van Harten, Gerard; Snik, Frans; Smit, Martijn; Stam, Daphne M.; Keller, Christoph U.; Laan, Erik C.; Verlaan, Adrianus L.; Vliegenthart, Willem A.; Ter Horst, Ramón; Navarro; Wielenga, Klaas (2011). Meynart, Roland; Neeck, Steven P.; Shimoda, Haruhisa, eds. "Spectropolarimeter for Planetary EXploration (SPEX): performance measurements with a prototype". Proceedings of the SPIE: Sensors, Systems, and Next-Generation Satellites XV 8176. pp. 81760D-81760D-12.

[3] van Harten, Gerard; Snik, Frans; Rietjens, Jeroen H. H.; Smit, J. Martijn; de Boer, Jozua; Diamantopoulou, Renia; Hasekamp, Otto P.; Stam, Daphne M.; Keller, Christoph U.; Laan, Erik C.; Verlaan, Ad L.; Vliegenthart, Willem A.; ter Horst, Rik; Navarro, Ramón; Wielinga, Klaas; Hannemann, Sandro; Moon, Scott G.; Voors, Robert (2011). Shaw, Joseph A.; Tyo, J. Scott, eds. "Prototyping for the Spectropolarimeter for Planetary EXploration (SPEX): calibration and sky measurements". Proceedings of the SPIE: Polarization Science and Remote Sensing V (Sunday 21 August 2011 San Diego, California, USA) 8160. pp. 81600Z. 